\title{
Bohumil Cenkl
}

L'équation de structure d'un espace à connexion projective

Czechoslovak Mathematical Journal, Vol. 14 (1964), No. 1, 79-94

Persistent URL: http://dml.cz/dmlcz/100602

\section{Terms of use:}

(C) Institute of Mathematics AS CR, 1964

Institute of Mathematics of the Czech Academy of Sciences provides access to digitized documents strictly for personal use. Each copy of any part of this document must contain these Terms of use.

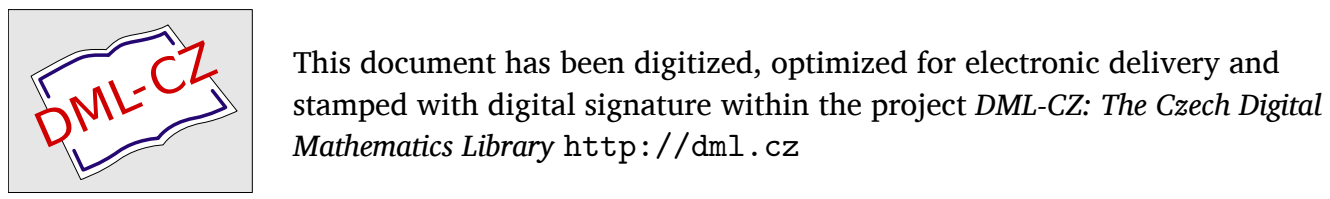




\title{
L'ÉQUATION DE STRUCTURE D'UN ESPACE À CONNEXION PROJECTIVE
}

\author{
Bohumil Cenkl, Praha
}

(Reçu le 17 février 1962)

\begin{abstract}
On trouve des conditions nécessaires et suffisantes pour qu'une surface dans un espace à connexion projective soit une surface de l'espace projectif. Ces conditions sont établies à l'aide d'objets associés aux points de la surface (p. ex. droites canoniques, quadriques canoniques, etc.).
\end{abstract}

On connaît bien les conditions nécessaires et suffisantes pour qu'une surface d'un espace à connexion projective soit une surface de l'espace projectif, telle p. ex. l'annulation du tenseur de courbure, ou bien d'autres modifications de cette condition analytique. Dans le présent travail, on donne des conditions géométriques qui sont nécessaires et suffisantes pour que la surface jouisse de la propriété en question. Il s'agit donc d'une caractéristique géométrique des conditions d'intégrabilité qui sont vérifiées pour une surface de l'espace projectif (voir p. ex. [5]). Par caractéristique géométrique nous entendons des conditions qu'on impose aux droites ou aux quadriques canoniques associées aux points de la surface considérée.

Le présent travail est divisé en trois parties. Dans la première partie, on définit la variété de König généralisée, à connexion projective (voir [6], [4]) et l'on donne une caractéristique géométrique du tenseur de courbure-torsion, sans connexion auxilliaire donnée sur la variété fondamentale, comme c'est le cas de [4]. Dans la deuxième partie on introduit un repère canonique associé aux points de la surface à connexion projective et l'on donne les définitions des objets géométriques les plus importants. La dernière partie contient enfin le théorème fondamental du travail entier.

\section{TENSEUR DE COURBURE DE LA VARIÉTÉ $P_{p n}^{N}$ A CONNEXION PROJECTIVE}

1. D’une manière tout à fait analogue à celle du travail [4], nous définissons la variété $P_{p n}^{N}(0 \leqq p \leqq N, 0<n ; p, n, N$ étant des entiers positifs $)$ de la façon suivante: Soit donnée une variété différentiable $X_{n}$ à $n$ dimensions, appelée variété fondamen- 
tale et supposons qu'on associe à chaque point $(\xi) \equiv\left(\xi^{1}, \ldots, \xi^{n}\right)$ de la variété $X_{n}$ un espace local $P_{N}(\xi)$ de dimension $N$, et, en celui-ci, un sous-espace $P_{p}(\xi)$ à $p$ dimensions (appelé centre de l'espace local). La connexion projective sur la variété ainsi définie soit donnée par les formes différentielles $\omega_{a}^{b}=\prod_{a x}^{b}(\xi) \mathrm{d} \xi^{\alpha}{ }^{1}$ ) par rapport au choix de la base $\left\{A_{0}(\xi), A_{1}(\xi), \ldots, A_{N}(\xi)\right\}$ dans les espaces locaux $P_{N}(\xi)$, telle que $\left\{A_{0}(\xi), A_{1}(\xi), \ldots, A_{p}(\xi)\right\}$ soit une base du centre $P_{p}(\xi)$ de l'espace local $P_{N}(\xi)$. (Les $A_{a}$ sont fonctions scalaires de $\xi^{\alpha}$.) Nous disons également que la connexion sur la variété $P_{p n}^{N}$ est donnée par les équations

$$
\mathrm{d} A_{a}=\omega_{a}^{b} A_{b}
$$

où nous avons une différentielle au premier membre dans le cas où nous considérons (1) suivant une courbe sur la variété fondamentale. Nous pouvons écrire les équations de structure de la variété $P_{p n}^{N}$ à connexion projective dans la forme

$$
\left[\mathrm{d} \omega_{a}^{b}\right]=\left[\omega_{a}^{c} \omega_{c}^{b}\right]-\Omega_{a}^{b} .
$$

Si nous avons choisi le repère

$$
\left\{A_{0}, A_{1}, \ldots, A_{N}\right\}
$$

dans l'espace local $P_{N}(\xi)$ de telle façon que

$$
\left\{A_{0}, A_{1}, \ldots, A_{p}\right\}
$$

soit le repère du centre $P_{p}(\xi)$, nous dirons que les transformations

$$
A_{a}=\sigma_{a}^{b^{\prime}} A_{b^{\prime}}, \quad \sigma_{\boldsymbol{A}}^{M^{\prime}}=0,
$$

sont des transformations admissibles de la base (3) si $\sigma_{a}^{b^{\prime}}$ sont fonctions scalaires de $(\xi)$ et si nous avons

$$
\sigma \equiv \operatorname{Det}\left|\sigma_{a}^{b}\right|=1, \quad \sigma_{A}^{M^{\prime}}=0 .
$$

Nous définnissons $\sigma_{a^{\prime}}^{b}$ par les équations

$$
\sigma_{a}^{b^{\prime}} \sigma_{c^{\prime}}^{a}=\delta_{c^{\prime}}^{b^{\prime}}, \quad \sigma_{a}^{b^{\prime}} \sigma_{b^{\prime}}^{c}=\delta_{a}^{c} .
$$

$\mathrm{Si}$

$$
E^{0}, E^{1}, \ldots, E^{N} ; \quad E^{a}=(-1)^{a}\left[A_{0}, \ldots, A_{a-1}, A_{a+1}, \ldots, A_{N}\right]
$$

et le repère de l'espace $P_{N}^{*}(\xi)$, dual de $P_{N}(\xi)$, nous avons évidemment

$$
E^{b}=\sigma_{a^{b}}^{b} E^{a^{\prime}} \text {. }
$$

$\left.{ }^{1}\right)$ Les indices prennent les valeurs suivantes:

$$
\begin{array}{ll}
a, b, \ldots=0,1, \ldots, N ; & A, B, \ldots=0,1, \ldots, p ; \\
\alpha, \beta, \ldots=1,2, \ldots, n ; & M, P, \ldots=p+1, \ldots, N .
\end{array}
$$


Les transformations (5) ne sont pas des transformations projectives générales; en effet, nous pouvons, pour les transformations projectives générales multiplier la matrice $\left\|\sigma_{a}^{b^{\prime}}\right\|$ par un facteur scalaire $f$ arbitraire.

Si ensuite

$$
\xi^{\alpha^{\prime}}=\xi^{\alpha^{\prime}}(\xi), \Delta=\operatorname{Det}\left|\frac{\partial \xi^{\alpha^{\prime}}}{\partial \xi^{\alpha}}\right| \neq 0,
$$

sont des transformations des paramètres sur la variété fondamentale $X_{n}$, nous pouvons définir un tenseur sur la variété $P_{p n}^{N}$ à connexion projective.

Nous appellons tenseur de caractéristique $\left(\begin{array}{lll}u & & s \\ & i & q \\ v & & t\end{array}\right)$ (cf. [4]) l'ensemble des fonctions $V_{\beta_{1}+\beta_{v} b_{1} \ldots b_{t}}^{\alpha_{1} \ldots \alpha_{u} a_{1} \ldots a_{s}}$, qui, lorsqu'on y applique les transformations (5), (10) ou qu'on les multiplie par un facteur scalaire $f$ ( $f$-transformations), se transforment suivant

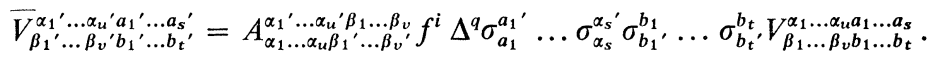

L'équation (1) fait voir que, par les transformations (5), (10) et les $f$-transformations, les fonctions $\Pi_{a x}^{b}(\xi)$ se transforment comme suit:

$$
\bar{\Pi}_{c^{\prime} \alpha^{\prime}}^{b^{\prime}}=\left\{\Pi_{c \alpha}^{b} \sigma_{c^{\prime}}^{c} \sigma_{b}^{b^{\prime}}-\partial_{\alpha} \sigma_{c}^{b^{\prime}} \sigma_{c^{\prime}}^{c}+\delta_{c}^{b} \sigma_{c^{\prime}}^{c} \sigma_{b}^{b^{\prime}} \partial_{\alpha} \log f\right\} A_{\alpha^{\prime}}^{\alpha} .
$$

Définissons maintenant des objets suivants

$$
\begin{gathered}
G_{a \alpha}^{b}=\Pi_{a \alpha}^{b}-(1 / N+1) \delta_{a}^{b} \Pi_{c \alpha}^{c}, \\
Q_{\alpha}=(1 / N+1) \Pi_{c \alpha}^{c} .
\end{gathered}
$$

Pour les transformations (5), (10), $f$, nous avons pour l'expression $\left(13_{1}\right)$

$$
G_{a^{\prime} \alpha^{\prime}}^{b^{\prime}}=\left\{G_{a \alpha}^{b} \sigma_{b}^{b^{\prime}}-\partial_{\alpha} \sigma_{a}^{b^{\prime}}\right\} A_{\alpha^{\prime}}^{\alpha} \sigma_{a^{\prime}}^{a},
$$

et nous voyons que $G_{a \alpha}^{b}$ est un $f$-invariant. Il est clair que l'expression

$$
R_{\alpha \beta a}^{b}=2 \partial_{[\alpha} G_{|a| \beta]}^{b}+2 G_{c[\alpha}^{b} G_{|a| \beta]}^{c}
$$

est un tenseur de caractéristique $\left(\begin{array}{l}0 \\ 2\end{array}, 0,0, \begin{array}{l}1 \\ 1\end{array}\right)$, appelé tenseur de courbure-torsion de la variété $P_{p n}^{N}$ à connexion projective; d'une manière analogue l'expression

$$
Q_{\alpha \beta}=2 \partial_{[\alpha} Q_{\beta]}
$$

est un tenseur de caractéristique $\left(\begin{array}{l}0 \\ 2\end{array}, 0,0\right)$.

Considérons encore le tenseur

$$
M_{\alpha \beta a}^{b}=2 \partial_{[\alpha} \Pi_{|a| \beta]}^{b}+2 \Pi_{a[\beta}^{c} \Pi_{|c| \alpha]}^{b}
$$

de caractéristique $\left(\begin{array}{ll}0 & , 0, \\ 2 & 1 \\ 2\end{array}\right)$. 
2. Dans le deuxième paragraphe de cette partie, nous allons donner la signification géométrique et les rapports qui existent entre les tenseurs (15), (16) et (17). Dans ce but, nous définissons d'abord la translation parallèle d'un point suivant une courbe donnée sur la variété fondamentale $X_{n}$. On sait bien (voir [6]), que la connexion de la variété, donnée par les équations (1), peut être décrite de la façon suivante: Sur la variété fondamentale $X_{n}$ soit donnée une courbe $\gamma$ par les équations paramétriques

$$
\xi^{\alpha}=\xi^{\alpha}(t),{ }^{1} t \leqq t \leqq{ }^{2} t ; \quad \xi^{\alpha}\left({ }^{1} t\right)={ }^{1} \xi^{\alpha}, \xi^{\alpha}\left({ }^{2} t\right)={ }^{2} \xi^{\alpha} .
$$

Considérons le système d'équations différentielles

$$
\frac{\mathrm{d} B_{a}}{\mathrm{~d} t}=\prod_{a \alpha}^{b}(\xi(t)) \frac{\mathrm{d} \xi^{\alpha}}{\mathrm{d} t} B_{b},
$$

dont la solution est un système monoparamétrique de repères dans l'espace local $P_{N}\left({ }^{1} \xi\right)$, à condition de prendre pour les conditions à l'origine des équations (19) les relations

$$
B_{a}\left({ }^{1} t\right)=A_{a}\left({ }^{1} \xi\right)
$$

où $\left\{A_{0}\left({ }^{1} \xi\right), \ldots, A_{N}\left({ }^{1} \xi\right)\right\}$ est la base de l'espace local $P_{N}\left({ }^{1} \xi\right)$. L'homographie $H_{\gamma}\left({ }^{1} \xi,{ }^{2} \xi\right)=H_{\gamma}$,

$$
H_{\gamma} A_{a}\left({ }^{2} \xi\right)=B_{a}\left({ }^{2} t\right)
$$

nous donne les rapports qui existent entre les espaces locaux projectifs $P_{N}\left({ }^{1} \xi\right), P_{N}\left({ }^{2} \xi\right)$, en fonction de la courbe $\gamma$.

Par translation parallèle d'un ensemble arbitraire $\mathfrak{M}$ de points de l'espace local $P_{N}\left({ }^{2} \xi\right)$ dans l'espace $P_{N}\left({ }^{1} \xi\right)$ suivant la courbe $\gamma$ nous entendons l'image $H_{\gamma} \mathfrak{M}$ de l'ensemble $\mathfrak{M}$ par l'homographie $\boldsymbol{H}_{\gamma}$. Fixons, sur la variété $X_{n}$, deux champs de vecteurs contravariants $u^{\alpha}, v^{\alpha}$ (tenseurs de caractéristique $\left(\begin{array}{l}1 \\ 0\end{array}, 0,0\right)$ ). Les trajectoires, du champ de vecteurs $u^{\alpha}(\xi)$ sont les courbes vérifiant les équations différentielles

$$
\frac{\mathrm{d} \eta^{\alpha}}{\mathrm{d} r}=u^{\alpha}(\eta)
$$

et, d'une façon analogue, nous obtenons les trajectoires du champ de vecteurs $v^{\alpha}(\xi$ ), en résolvant les équations

$$
\frac{\mathrm{d} \eta^{\alpha}}{\mathrm{d} s}=v^{\alpha}(\eta)
$$

La solution du système (23) correspondant aux conditions initiales $\eta^{\alpha}=\xi^{\alpha}$ pour $s=0$, ( $\xi^{\alpha}$ étant un point choisi arbitrair ement sur la variété ondamentale $X_{n}$ ) peut être écrite sous la forme

$$
\eta^{\alpha}=\xi^{\alpha}+s v^{\alpha}+\frac{s^{2}}{2 !} v^{\beta} \partial_{\beta} v^{\alpha}+\frac{s^{3}}{3 !}\left(v^{\beta} \partial_{\beta}\right)^{2} v^{\alpha}+\ldots=\exp \underset{v}{(s X)} \xi^{\alpha},
$$


où $X$ est l'opérateur $X=v^{\alpha} \partial_{\alpha}$. Nous obtenons ainsi une trajectoire du champ vectoriel $v^{\alpha}(\xi)$; nous la désignerons par $\gamma_{1}$. Sur la courbe $\gamma_{1}$, considérons le point $\eta_{1}^{\alpha}$ correspondant à la valeur $-s$ du paramètre. Nous avons donc

$$
\eta_{1}^{\alpha}=\xi^{\alpha}-s v^{\alpha}+\frac{s^{2}}{2 !} v^{\beta} \partial_{\beta} v^{\alpha}-\frac{\beta^{3}}{3 !}\left(v^{\beta} \partial_{\beta}\right)^{2} v^{\alpha}+\ldots=\exp (-\underset{v}{s} X) \xi^{\alpha} .
$$

D'une manière analogue, nous pouvons écrire la trajectoire $\gamma_{2}$ du champ vectoriel $u^{\alpha}(\xi)$ passant par le point $(\xi)$, donc la solution du système (22) correspondant aux conditions initiales $\eta^{\alpha}=\xi^{\alpha}$ pour $r=0$, sous la forme

$$
\eta^{\alpha}=\xi^{\alpha}+r u^{\alpha}+\frac{r^{2}}{2 !} u^{\beta} \partial_{\beta} u^{\alpha}+\frac{r^{3}}{3 !}\left(u^{\beta} \partial_{\beta}\right)^{2} u^{\alpha}+\ldots=\exp \underset{u}{(r X) \xi^{\alpha}} .
$$

Par translation parallèle du repère $A_{a}\left(\eta_{1}^{\alpha}\right)$ du point $\left(\eta_{1}\right)$ au point $(\xi)$ de la variété fondamentale $X_{n}$ suivant la courbe $\gamma_{1}$, nous obtenons dans l'espace local $P_{N}(\xi)$ le repère ${ }^{1} B_{a}(\xi)=B_{a}(-s)$, où $B_{a}$ est la solution du système (19) où nous écrivons $t$ au lieu de $s$ (dans l'espace local $P_{N}(\xi)$ ) correspondant aux conditions à l'origine $B_{a}(0)=A_{a}(\xi)$. Nous avons donc

$$
\begin{aligned}
B_{a}(\xi)=\left\{\delta_{a}^{b}\right. & -s v^{\alpha} \Pi_{a \alpha}^{b}+\frac{s^{2}}{2 !}\left(\partial_{\beta} \Pi_{a \alpha}^{b} v^{\alpha} v^{\beta}+\Pi_{a \alpha}^{b} v^{\beta} \partial_{\beta} v^{\alpha}+\right. \\
& \left.\left.+\Pi_{a \alpha}^{c} \Pi_{c \beta}^{b} v^{\alpha} v^{\beta}\right)+\ldots\right\} A_{b}(\xi),
\end{aligned}
$$

(où l'expression $\{\ldots\}$ est considéré, bien entendu, au point $(\xi)$ de la variété $X_{n}$ ). L'équation (25) nous détermine en réalité un opérateur $R$ qui associe à chaque repère $A_{a}(\xi)$ au point $(\xi)$ de la variété $X_{u}$ un repère ${ }^{1} B_{a}(\xi)$ du même espace local $P_{N}(\xi)$ et que nous obtenons par translation parallèle suivant la trajectoire du champ vectoriel $v^{\alpha}(\xi)$, translation correspondante à la valeur $s$ du paramètre sur la trajectoire de ce champ. Nous noterons donc

$$
\stackrel{s v}{R} A_{a}(\xi)=\stackrel{1}{B_{a}}(\xi)
$$

Cherchons maintenant à l'aide de $(19),(25)$ le repère ${ }^{r u}{ }^{* v} R_{a}(\xi)=\stackrel{r u}{R} B_{c}(\xi)$, c'est-à-dire, dans l'espace local $P_{N}(\xi)$, le repère ${ }^{2}(\xi)$ que l'on obtient par translation parallèle du repère $B_{a}(\xi)$ du point

$$
\eta_{2}^{\alpha}=\xi^{\alpha}-r u^{\alpha}+\frac{r^{2}}{2 !} u^{\beta} \partial_{\beta} u^{\alpha}-\ldots=\exp \left(-\underset{u}{r X)} \xi^{\alpha}\right.
$$

de la trajectoire $\gamma_{2}$ du champ vectoriel $u^{\alpha}(\xi)$ suivant la courbe $\gamma_{2}$ au point $\xi_{2}$. A l'aide 
des équations (19), (25), nous obtenons d'une manière tout à fait analogue au procédé de ci-dessus

$$
\begin{gathered}
\stackrel{2}{B_{a}}(\xi)=\left\{\delta_{a}^{b}-r \Pi_{\alpha \beta}^{b} u^{\beta}-s v^{\alpha} \Pi_{a \alpha}^{b}+r s\left(u^{\beta} \partial_{\beta} v^{\alpha} \Pi_{a \alpha}^{b}+\right.\right. \\
\left.+v^{\alpha} u^{\beta} \partial_{\beta} \Pi_{a \alpha}^{b}+v^{\alpha} u^{\beta} \Pi_{a \alpha}^{c} \Pi_{c \beta}^{b}\right)+\frac{s^{2}}{2 !}\left(\partial_{s} \Pi_{a \alpha}^{b} v^{\alpha} v^{\beta}+\Pi_{a \alpha}^{b} v^{\beta} \partial_{\beta} v^{\alpha}+\Pi_{a \alpha}^{c} \Pi_{c \beta}^{b} v^{\alpha} v^{\beta}\right)+ \\
\left.+\frac{r^{2}}{2 !}\left(\partial_{\alpha} \Pi_{a \beta}^{b} u^{\alpha} u^{\beta}+\Pi_{a \beta}^{c} \Pi_{c \alpha}^{b} u^{\alpha} u^{\beta}+\Pi_{a \beta}^{b} u^{\alpha} \partial_{\alpha} v^{\beta}\right)+\ldots\right\} A_{b}(\xi) .
\end{gathered}
$$

Si nous calculons d'une façon analogue le repère $\stackrel{-s v}{R} \stackrel{r u}{R} \stackrel{1}{B_{a}}(\xi)=\stackrel{3}{B_{a}}(\xi)$ et le repère

$\stackrel{-r u}{R} B_{a}(\xi)=\stackrel{*}{B_{a}}(\xi)$, nous obtenons, comme il est aisé de le voir,

$$
\stackrel{*}{B_{a}}(\xi)=A_{a}(\xi)-r s u^{\beta} v^{\alpha} M_{\alpha \beta a}^{b} A_{b}(\xi),
$$

où $M_{\alpha \beta a}^{b}$ est le tenseur défini par l'équation (17).

Les équations (13), (15), (16) et (17) font voir que l'on a

$$
R_{\alpha \beta a}^{b}=M_{\alpha \beta a}^{b}-\delta_{a}^{b} Q_{\alpha \beta} .
$$

Au lieu de (29) nous pouvons donc écrire

$$
\stackrel{*}{B_{a}}(\xi)=A_{a}(\xi)\left(1-Q_{\alpha \beta} r s u^{\beta} v^{\alpha}\right)-r s u^{\beta} v^{\alpha} R_{\alpha \beta a}^{b} A_{b}(\xi) .
$$

Si $u^{a}(\xi)$ est un point contravariant (un tenseur de caractéristique $\left(\begin{array}{ll}1 & 0 \\ & 0\end{array}\right)$ ) défini sur la variété $P_{p n}^{N}$, alors en vertu de (31) nous avons

$$
\stackrel{*}{u}^{a}(\xi)=u^{a}(\xi)\left(1-Q_{\alpha \beta} r s u^{\beta} v^{\alpha}\right)-r s u^{\beta} v^{\alpha} R_{\alpha \beta b}^{a} u^{b}(\xi),
$$

ce qui est une expression qui a été déjà trouvée dans le travail [4], mais sous l'hypothèse d'une connexion auxilliaire donnée sur la variété $X_{n}$. La signification géométrique de l'annulation du tenseur de courbure-torsion $R_{\alpha \beta a}^{b}$ et du tenseur de torsion $R_{\alpha \beta A}^{M}$, qui découle immédiatement de (32), est contenu dans le travail [4].

Si nous écrivons les équations de structure (2) d'une façon plus détaillée (en y substituant $\omega_{a}^{b}=\Pi_{a \alpha}^{b} \mathrm{~d} \xi^{\alpha}$ ) et que nous utilisions (17), nous voyons que l'on a

$$
\Omega_{a}^{b}=M_{\alpha \beta a}^{b}\left[\mathrm{~d} \xi^{\alpha} \mathrm{d} \xi^{\beta}\right]
$$

\section{LE REPÉRE CANONIQUE DE LA SURFACE $P_{02}^{3}$}

1. Nous appellerons la variété $P_{02}^{3}$ à connexion projective, prise au sens du paragraphe précédent, surface $\pi$ à connexion projective de dimension 3 , soit d'une façon plus concise: surface $\pi$. Nous dénoterons $\pi$ la variété fondamentale $X_{2}$ et nous parle- 
rons dans le même sens d'une surface $\pi$. Les paramètres pricipaux sur la surface $\pi$ seront notés $u, v$ au lieu de $\xi^{1}, \xi^{2}$.

Soit donné, dans l'espace local $P_{3}(\xi)$ le repère

$$
\left\{A_{0}, A_{1}, A_{2}, A_{3}\right\}
$$

tel que le point $A_{0}$, centre de l'espace local, soit un point de la variété fondamentale $\pi$ et que l'on ait (cf. [6], [3])

$$
\begin{aligned}
& \mathrm{d} A_{0}=\omega_{0}^{0} A_{0}+\mathrm{d} u A_{1}+\mathrm{d} v A_{2}, \\
& \mathrm{~d} A_{1}=\omega_{1}^{0} A_{0}+\omega_{1}^{1} A_{1}+\beta \mathrm{d} u A_{2}+(1-h) \mathrm{d} v A_{3}, \\
& \mathrm{~d} A_{2}=\omega_{2}^{0} A_{0}+\gamma \mathrm{d} v A_{1}+\omega_{2}^{2} A_{2}+(1+h) \mathrm{d} u A_{3}, \\
& \mathrm{~d} A_{3}=\omega_{3}^{0} A_{0}+\omega_{3}^{1} A_{1}+\omega_{3}^{2} A_{2}+\omega_{3}^{3} A_{3}, \\
& \omega_{0}^{0}+\omega_{1}^{1}+\omega_{2}^{2}+\omega_{3}^{3}=0, \\
& \omega_{k}^{i}=a_{k}^{i} \mathrm{~d} u+b_{k}^{i} \mathrm{~d} v, \quad(i, k=0,1,2,3), \\
& a=a_{0}^{0}-a_{1}^{1}-a_{2}^{2}+a_{3}^{3}, \quad b=b_{0}^{0}-b_{1}^{1}-b_{2}^{2}+b_{3}^{3} .
\end{aligned}
$$

Nous appellons transformations admissibles les transformations de la base et des paramètres locaux qui transforment la matrice $\omega_{i}^{k}$; en une matrice du même type que (1.2), c'est-à-dire les transformations qui conservent la spécialisation du repère exprimée par les équations (1.2). Il est évident qu'elles sont données par les équations suivantes

$$
\begin{aligned}
u & =u(\bar{u}), \quad v=v(\bar{v}), \quad\left(r=u^{\prime}=\mathrm{d} u / \mathrm{d} \bar{u}, s=v^{\prime}=\mathrm{d} v / \mathrm{d} \bar{v}\right) . \\
A_{0} & =\alpha_{0}^{0} \bar{A}_{0}, \quad A_{1}=\alpha_{1}^{0} \bar{A}_{0}+r^{-1} \alpha_{0}^{0} \bar{A}_{1}, \quad A_{2}=\alpha_{2}^{0} \bar{A}_{0}+s^{-1} \alpha_{0}^{0} \bar{A}_{2}, \\
A_{3} & =\alpha_{3}^{0} \bar{A}_{0}+\alpha_{3}^{1} \bar{A}_{1}+\alpha_{3}^{2} \bar{A}_{2}+r^{-1} s^{-1} \alpha_{0}^{0} \bar{A}_{3}, \quad\left(\alpha_{0}^{0}\right)^{4}=r^{2} s^{2}, \\
\alpha_{1}^{0} & =(1-h) s \alpha_{3}^{2}, \quad \alpha_{2}^{0}=(1+h) r \alpha_{3}^{1},
\end{aligned}
$$

où $\alpha_{i}^{k}$ sont des fonctions arbitraires des paramètres $u, v$ sur la surface $\pi$.

Les homographies $K$ de l'espace local $P_{3}(\xi)$ dans lui-même qui conservent l'élément du troisième ordre du développement ${ }^{*}$ de la surface $\pi$ (du développement de toutes les courḅes de la surface $\pi$ passant par le point $(\xi)$ ), c'est-à-dire les homographies $\boldsymbol{K}$ telles que ${ }^{*}$ et $\boldsymbol{K}_{\pi}^{*}$ ont un contact géométrique du troisième ordre sont de deux types différents. Les homographies du premier type conservent les tangentes asymptotiques, tandis que les homographies du deuxième type associent l'une des tangetes asymptotiques à l'autre et vice versa. Nous pouvons écrire les homographies du premier type, le repère étant fixe de la manière donnée ci-dessus, sous la forme suivante (cf. [2])

$$
\begin{aligned}
& K A_{0}=A_{0}, \\
& K A_{1}=\left\{\alpha_{32} \varepsilon+\frac{1}{3} h \alpha_{32} \varepsilon+\frac{1}{3} a(\varepsilon-1)\right\} A_{0}+\varepsilon A_{1}, \\
& \kappa A_{2}=\left\{\alpha_{31} \varepsilon^{2}-\frac{1}{3} h \alpha_{31} \varepsilon^{2}+\frac{1}{3} b\left(\varepsilon^{2}-1\right)\right\} A_{0}+\varepsilon^{2} A_{2}, \\
& \kappa A_{3}=\alpha_{30} A_{0}+\alpha_{31} A_{1}+\alpha_{32} A_{2}+A_{3}
\end{aligned}
$$


Il est aisé de voir que l'on a: L'homographie générale $\boldsymbol{K}$ conservant l'élément $d u$ troisième ordre de la surface $\pi$ (nous entendons par cela le développement ${ }^{*}$ dans l'espace local) n'est pas une transformation admissible. Si nous avons $h=0$ (surface sans torsion), alors $a=b=0$ est la condition nécessaire et suffisante pour que $\boldsymbol{K}$ soit une transformation admissible (5).

Nous savons que $h=0$ est la condition nécessaire et suffisante pour qu'il y ait sur la surface $\pi$ un réseau réciproquement conjugué, c'est-à-dire un réseau de courbes tel que les tangentes aux courbes d'une couche suivant une courbe de l'autre couche forment une surface développable et vice versa (cf. [6]).

Considérons maintenant sur la surface $\pi$ une courbe $\gamma: v=v(u)$, tangente au point $A_{0}$ à une des asymptotiques, p.ex. $v=$ const. L'invariant de contact de SmithMehmke de ces deux courbes est alors $1+v=\left(\beta+v^{\prime \prime}\right) / \beta$. En chaque point de la courbe $\gamma$ considérons les tangentes aux asymptotiques $u=$ const. Désignons par $Q_{v}(v)$ une quadrique qui a un contact de second ordre avec la surface réglée qui vient d'être définie. Nous pouvons écrire l'équation de la quadrique $Q_{v}(v)$ sous la forme (cf. [9])

$$
\begin{gathered}
(1+h) x^{1} x^{2}-x^{0} x^{3}+\frac{1}{2}[a+h u /(1+h)] x^{1} x^{3}=\frac{1}{2}(1+h)^{-1} . \\
\cdot\left(1+h a_{3}^{1}-a_{2}^{0}+v \beta \gamma\right)\left(x^{3}\right)^{2} .
\end{gathered}
$$

L'équation de la quadrique analogue $Q_{u}(v)$ (nous changeons seulement les asymptotiques) sera

$$
\begin{gathered}
(1-h) x^{1} x^{2}-x^{0} x^{3}+\frac{1}{2}[b-h v /(1-h)] x^{2} x^{3}= \\
\quad=\frac{1}{2}(1-h)^{-1}\left[(1-h) b_{3}^{2}-b_{1}^{0}+v \beta \gamma\right]\left(x^{3}\right)^{2} .
\end{gathered}
$$

Nous avons donc un faisceau $Q(v, \lambda)$ de quadriques de Darboux. Le faisceau $Q(v, 1)$ s'appelle faisceau de Darboux principal et la quadrique $Q(0,1)$ dont l'équation est

$$
\begin{gathered}
x^{1} x^{2}-x^{0} x^{3}+\frac{1}{4}[a+h u /(1+h)] x^{1} x^{3}+\frac{1}{4}[b-h v /(1-h)] x^{2} x^{3}= \\
=\frac{1}{4}\left[a_{3}^{1}+b_{3}^{2}-a_{2}^{0} /(1+h)-b_{1}^{0} /(1-h)\right]\left(x^{3}\right)^{2},
\end{gathered}
$$

s'appelle quadrique principale de Lie. Dans le faisceau $Q(v, \lambda)$, il existe un faisceau à un paramètre de quadriques singulières, composées chacune de deux plans $\Pi_{1}, \Pi_{2}(v)$, où $\Pi_{1}$ est le plan tangent à la surface au point considéré $A_{0}$. Si $h=0$, alors $a=b=0$ est la condition nécessaire et suffisante pour que le plan $\Pi_{2}$ coïncide avec $\Pi_{1}$. Pour une caractéristique géométrique plus détaillée de l'égalité $a=b=0$ voir p.ex. [3]

A chaque point de la surface $\pi$ on peut associer un faisceau à trois paramètres de quadriques qui ont avec la surface un contact analytique du second ordre. Leur équation est (voir [9])

$$
x^{0} x^{3}-x^{1} x^{2}-c_{13} x^{1} x^{3}-c_{23} x^{2} x^{3}=\frac{1}{2} c_{33}\left(x^{3}\right)^{2},
$$

où $c_{13}, c_{23}, c_{33}$ sont des fonctions arbitraires du point $(\xi)$ de la surface $\pi$. 
Remarque. Toutes les constructions figurant dans les définitions précédentes doivent être faites dans le développement dans l'espace local du point correspondant.

Parmi les quadriques (10), il est possible de caractériser géométriquement un système monoparamétrique de quadriques (ce sont les quadriques essentielles de Darboux):

$$
x^{0} x^{3}-x^{1} x^{2}=\frac{1}{2} c_{33}\left(x^{3}\right)^{2} .
$$

Cela se fait de la façon suivante: Par le point $A_{0}$ de la surface $\pi$ faisons passer une droite $p$ qui n'est pas située dans le plan tangent. Soit $R_{1}$ la surface réglée formée des tangentes aux asymptotiques $v=$ const suivant l'asymptotique $u=$ const; soit $R_{2}$ la surface définie d'une manière analogue lorsqu'on échange les rôles joués par les asymptotiques. Soit $r_{1}$ la droite sur $R_{1}$ passant par le point $A_{0}$ de la surface $\pi$, soit $r_{2}$ la droite analogue sur $R_{2}$. Les plans déterminés par les droites $p, r_{1}$, ou $p, r_{2}$ touchent la surface non-développable $R_{1}$, ou $R_{2}$ resp., au point $P_{1}$, ou $P_{2}$ resp. Nous désignerons par $q$ la droite passant par les points $P_{1}, P_{2}$ et nous l'appellerons droite associée à la droite $p$. La quadrique (11) est une quadrique osculatrice et telle que $p$ et $q$ sont polaires conjuguées par rapport à elle (cf. [3]).

2. Par arrête de Green I associée à la surface $\pi$ à connexion projective (abbrev. GI) nous entendrons la droite associée au point de la surface $\pi$, située hors du plan tangent en ce point, définie comme suit: Soit $p$ une droite quelconque passant par le point $A_{0}$ de la surface $\pi$, située hors du plan tangent $\tau$ au point $A_{0}$. Prenons un point arbitraire de la droite $p$, différent de $A_{0}$, et projetons-en les courbes asymptotiques de la surface $\pi$ dans le plan tangent $\tau$. Construisons des sections coniques qui ont, au point $A_{0}$, un contact du troisième ordre avec la projection d'une des asymptotiques. Construisons ensuite les pôles des tangentes asymptotiques par rapport aux coniques ainsi definies. En joignant ces pôles, nous obtenons à côté des tangentes asymptotiques encore une droite $p^{\prime}$ dans $\tau$, et qui ne passe pas par $A_{0}$. Si les droites $p, p^{\prime}$ sont conjuguées polaires par rapport aux quadriques essentielles de Darboux (11), alors la droite $p$ sera appelée arrête du Green (GI). Nous l'obtenons comme la droite de jonction des points

$$
\begin{gathered}
{\left[A_{0},(4+h)^{-1}\left(b_{0}^{0} b_{1}^{1}-2 b_{2}^{2}+\partial \log \gamma / \partial v\right) A_{1}+\right.} \\
\left.+(4-h)^{-1}\left(a_{0}^{0}+a_{2}^{2}-2 a_{1}^{1}+\partial \log \beta / \partial u\right) A_{2}+A_{3}\right] .
\end{gathered}
$$

La droite qui lui est polaire par rapport à (11), c'est

$$
\begin{gathered}
{\left[A_{1}+(4+h)^{-1}\left(a_{0}^{0}+a_{2}^{2}-2 a_{1}^{1}+\partial \log \beta / \partial u\right) A_{0}, A_{2}+\right.} \\
\left.+(4-h)^{-1}\left(b_{0}^{0}+b_{1}^{1}-2 b_{2}^{2}+\partial \log \gamma / \partial v\right) A_{0}\right] .
\end{gathered}
$$

Toutes les quadriques mentionnées dans notre travail, à l'exception de la quadrique singulière du faisceau $Q(v, \lambda)$, sont des généralisations des quadriques associées aux points d'une surface dans l'espace projectif. Il en est de même de la droite GI. Il est évident que nous obtenons les arrêtes de Green, lorsque les droites $p, p^{\prime}$ sont 
conjuguées polaires par rapport à une des quadriques mentionnées ci-dessus. Dans notre cas, nous avons choisi la quadrique essentielle de Darboux, car les droites $p, p^{\prime}$ conjuguées polaires par rapport à elle sont aussi associées (la droite $p^{\prime}$ est associée à la droite $p$ ).

Sur la surface $\pi$, donnée par les équations (12) et que nous supposons sans torsion $(h=0)$, soit donnée une asymptotique $v=$ const. Construisons les tangentes à cette asymptotique et cherchons un complexe linéaire réglé $k_{1}$ qui ait un contact réglé du quatrième ordre avec la surface réglée ainsi définie. Nous obtenons un complexe analogue $k_{2}$ en prenant l'asymptotique $u=$ const. Dans le iaisceau déterminé par les complexes $k_{1}, k_{2}$ il y a des complexes linéaires spéciaux dont les axes sont des directrices de Wilczynski (cf. [9])

où

$$
\begin{aligned}
& {\left[A_{0},-\frac{1}{2}\left(\alpha / \beta+\frac{1}{2} b\right) A_{1}-\frac{1}{2}\left(\alpha_{2} / \gamma+\frac{1}{2} a\right) A_{2}+A_{3}\right],} \\
& {\left[A_{1}-\frac{1}{2}\left(\alpha_{2} / \gamma-\frac{1}{2} a\right) A_{0}, A_{2}-\frac{1}{2}\left(\alpha_{1} / \beta-\frac{1}{2} b\right) A_{0}\right],}
\end{aligned}
$$

$$
\begin{aligned}
& \alpha_{1}=a_{3}^{2}-a_{1}^{0}-\frac{1}{2} a_{u}+\frac{1}{2} a\left(a_{3}^{3}-a_{2}^{2}\right)-\frac{1}{4} a^{2}, \\
& \alpha_{2}=b_{3}^{1}-b_{2}^{0}-\frac{1}{2} b_{v}+\frac{1}{2} b\left(b_{3}^{3}-b_{1}^{1}\right)-\frac{1}{4} b^{2} .
\end{aligned}
$$

3. Supposons que le repère sur la surface $\pi$ soit spécialisé de telle façon que l'on ait (1.2). A présent, nous allons étudier les enveloppes de plans osculateurs de certains systèmes de courbes sur la surface. Ayons donc sur la surface $\pi$ une courbe donnée par

$$
v=\varphi(u), \quad\left(m=\mathrm{d} v / \mathrm{d} u, n=\mathrm{d}^{2} v / \mathrm{d} u^{2}\right) .
$$

Le plan osculateur de cette courbe est déterminé par l'équation

$\left[A_{0}, A_{1}+m A_{2},\left(\beta+n+m\left(a_{2}^{2}-a_{1}^{1}\right)+m^{2}\left(b_{2}^{2}-b_{1}^{1}\right)-m^{3} \gamma\right) A_{2}+2 m A_{3}\right]=0$, ou, en coordonnées locales,

$$
\left(\beta+n-m^{3} \gamma+m\left(a_{2}^{2}-a_{1}^{1}\right)+m^{2}\left(b_{2}^{2}-b_{1}^{1}\right) x^{3}+2 m^{2} x^{1}-2 m x^{2}=0 .\right.
$$

Le plan donné par l'equation (17) dépend évidemment de deux paramètres $m, n$. Soient données deux fonctions scalaires $m_{1}(h), m_{2}(h)$ de la torsion $h$ de la surface $\pi$ et telles que. Alors la forme

$$
\Psi_{m_{1} m_{2}}=m_{1} \beta \mathrm{d} u^{3}+m_{2} \gamma \mathrm{d} v^{3}
$$

se transforme par des transformations admissibles comme suit

$$
\bar{\Psi}_{\bar{m}_{1}, \bar{m}_{2}}=\Psi_{m_{1}, m_{2}} 1 /\left(u^{\prime}, v^{\prime}\right), \quad\left(\bar{m}_{1}=m_{1}, \bar{m}_{2}=m_{2}\right) .
$$

L'annulation de la forme $\Psi_{m_{1}, m_{2}}$ donne l'équation différentielle des courbes sur la surface $\pi$ qui sont des courbes de Darboux généralisées (pour $1=m_{1}(h)=m_{2}(h)$ 
voir p. ex. [6], pour $m_{1}(h)=1-h, m_{2}(h)=-(1+h)$ voir [1], pour $m_{1}(h)=$ $=(1-h)^{2}, m_{2}(h)=(1+h)^{2}$ voir [7]). Dans la suite, nous noterons

$$
m_{1} \beta=B, \quad m_{2} \gamma=C .
$$

Nous pouvons donc donner les tangentes - $D$ (tangentes aux courbes de Darboux généralisées au point considéré) par les équations

$$
y=\varepsilon \sqrt[3]{ }(B / C) x, \quad y=\varepsilon^{2} \sqrt[3]{(B / C) x,} \quad y=\varepsilon^{3} \sqrt[3]{(B / C) x}
$$

où $\varepsilon$ désigne la racine cubique de $(-1)$.

Soit maintenant (15) une courbe telle que les tangentes (21) et la tangente à (15) ont le birapport constant:

$$
\left(m, \varepsilon(B / C)^{1 / 3}, \varepsilon^{2}(B / C)^{1 / 3}, \varepsilon^{3}(B / C)^{1 / 3}\right)=\text { const . }
$$

Alors, nous avons $m=\lambda(B / C)^{1 / 3}, \lambda$ étant une constante arbitraire. Nous avons donc

$$
n=\frac{1}{3} m \frac{\partial}{\partial u}\left(\log \frac{B}{C}\right)+\frac{1}{3} m^{2} \frac{\partial}{\partial v}\left(\log \frac{B}{C}\right) .
$$

Le plan osculateur d'une courbe d'un tel système est donné par l'équation

$$
\begin{aligned}
& m^{3} \gamma z-2 m^{2}\left\{x+\frac{1}{2}\left(b_{2}^{2}-b_{1}^{1}\right) z+\frac{1}{6} z \partial \log (B / C) / \partial v\right\}+ \\
& +2 m\left\{y+\frac{1}{2}\left(a_{1}^{1}-a_{2}^{2}\right) z-\frac{1}{6} z \partial \log (B / C) / \partial u\right\}-\beta z=0 .
\end{aligned}
$$

Par chaque point de l'espace local passent exactement trois plans qui sont plans osculateurs des courbes du système considéré. Ces plans enveloppent un cône dont nous obtenons les plans tangents stationnaires en éliminant $x, y, z$ de l'équation (24) et des deux équations que nous obtenons en différentiant (24) deux fois de suite par rapport à $m$. Il en vient

$$
m^{3} C=B \text {. }
$$

Les trois plans tangents stationnaires coupent le plan tangent les tangentes de Segre genéralisées et passent tous par une droite

$$
x=\frac{1}{2}\left(b_{1}^{1}-b_{2}^{2}-\frac{1}{3} \partial \log (B / C) / \partial v\right) z, \quad y=\frac{1}{2}\left(a_{2}^{2}-a_{1}^{1}+\frac{1}{3} \partial \log (B / C) / \partial u\right) z,
$$

que nous appellons axe de Čech de la surface $\pi$ à connexion projective. Il existe tout un système de ces droites, dépendant du choix des fonctions $m_{1}, m_{2}$.

Il est aisé de voir que la forme

$$
\varphi=B C \mathrm{~d} u \mathrm{~d} v
$$

est invariante par les transformations (4), (5). Nous appellons géodésiques et nous notons $g$, les courbes (15) pour lesquelles l'intégrale

$$
\int(2 B C \mathrm{~d} u \mathrm{~d} v)^{1 / 2}=\int\left(2 B C_{m}\right)^{1 / 2} \mathrm{~d} u
$$

prend sa valeur extrême. 
Les équations d'Euler-Lagrange sont donc

$$
n=m \partial \log B C / \partial u-m^{2} \partial \log B C / \partial v .
$$

Les plans osculateurs de ces géodésiques sont donnés par l'équation

$$
\begin{aligned}
& m^{3} \gamma z-2 m^{2}\left\{x+\frac{1}{2}\left(b_{2}^{2}-b_{1}^{1}\right) z-\frac{1}{2} z \partial \log B C / \partial v\right\}+ \\
& +2 m\left\{y+\frac{1}{2}\left(a_{1}^{1}-a_{2}^{2}\right) z-\frac{1}{2} z \partial \log B C / \partial u\right\}-\beta z=0 .
\end{aligned}
$$

Il passe de nouveau par chaque point de l'espace local trois plans qui sont plans osculateurs des géodésiques $g$. Ces plans enveloppent un cône qui a trois plans tangents stationnaires dont la droite d'intersection avec le plan tangent est de nouveau donnée par l'équation (25). Les plans tangents stationnaires se coupent en une droite,

$$
x=\frac{1}{2}\left(b_{1}^{1}-b_{2}^{2}+\partial \log B C / \partial v\right) z, \quad y=\frac{1}{2}\left(a_{2}^{2}-a_{1}^{1}+\partial \log B C / \partial u\right) z,
$$

que nous appellons normale de Fubini de la surface $\pi$ à connexion projective. Les droites (26), (31) sont deux droites différentes passant par le point $A_{0}$ de la surface $\pi$. Elles déterminent donc un plan, formé de droites (dépendant du paramètre $\mu$ )

$$
\begin{aligned}
& x=\frac{1}{2}\left(b_{1}^{1}-b_{2}^{2}-\partial \log B / \partial v+\mu \partial \log B^{2} C / \partial v\right) z, \\
& y=\frac{1}{2}\left(a_{2}^{2}-a_{1}^{1}-\partial \log C / \partial u+\mu \partial \log B C^{2} / \partial u\right) z .
\end{aligned}
$$

Le plan formé des droites (32) s'appelle plan canonique. Dans ce plan, nous définissons les droites suivantes (p.ex. à l'aide du birapport à l'axe de Čech, à la normale de Fubini, ou à la tangente canonique) pour différentes valeurs de $\mu$ :

$$
\begin{aligned}
\mu & =0-\text { directrice de Wilczynski; } & \mu & =\frac{1}{4}-\text { droite de Cartan; } \\
\mu & =\frac{1}{3}-\text { axe de Čech; } & \mu & =\frac{1}{2}-\text { arrête de Green II; } \\
\mu & =1-\text { normale de Fubini; } & \mu & =\infty \text { tangente canonique. }
\end{aligned}
$$

Choisissons maintenant le point $A_{3}$ du repère (1) sur la droite (32) (pour une certaine valeur du paramètre $\mu$ ) et en même temps sur une des quadriques invariantes; p.ex. sur la quadrique principale de Lie $Q(0,1)$. Il faut donc que l'on ait

$$
\begin{aligned}
b_{1}^{1}-b_{2}^{2}-\partial \log B / \partial v+\mu \partial \log B^{2} C / \partial v & =0, \\
a_{2}^{2}-a_{1}^{1}-\partial \log C / \partial u+\mu \partial \log B C^{2} / \partial u & =0, \\
a_{3}^{1}+b_{3}^{2}-a_{2}^{0} /(1+h)-b_{1}^{0} /(1-h) & =0 .
\end{aligned}
$$

De cette façon, nous avons déterminé sur la surface $\pi$ à connexion projective le repère canonique qui est une généralisation du repère pour une surface dans l'espace projectif. La normalisation des points $A_{i}$ ne dépénd que des transformations (4). Les points géométriques $A_{i}$ sont déterminés d'une façon univoque. 


\section{CONDITIONS D'INTÉGRABILITÉ POUR UNE SURFACE \\ DANS L'ESPACE PROJECTIF}

1. Dans cette partie de notre travail, nous allons donner une caractéristique géométrique des conditions d'intégrabilité du système (1.1), c'est-à-dire une caractéristique géométrique de l'annulation des formes $\Omega_{a}^{b}$. Dans la suite, nous exprimerons les conditions nécessaires et suffisantes pour que deux objets géométriques coincident, ou qu'il aient une certaine propriété. A chaque pas, c'est-à-dire en formulant de nouvelles conditions, nous supposons toujours que les conditions précédentes sont satisfaites.

Supposons donc que nous ayons sur la surface $\pi$ le repère (2.2) et que (2.3) ait lieu. Supposons de plus,

$$
h=a=b=0
$$

la signification géométrique de ces égalités a été donnée déjà. Pour que les droites GI et GII soient identiques, il faut et il suffit que l'on ait

$$
a_{0}^{0}-a_{2}^{2}=0, \quad b_{0}^{0}-b_{1}^{1}=0 .
$$

En vertu des équations (2.3), (1), (2), nous pouvons donc écrire

$$
\tilde{a}=a_{0}^{0}=-a_{1}^{1}=a_{2}^{2}=-a_{3}^{3}, \quad \tilde{b}=b_{0}^{0}=b_{1}^{1}=-b_{2}^{2}=-b_{3}^{3} .
$$

Pour que la directrice de Wilczynski, définie comme sommet d'un certain complexe linéaire spécial (c'est la droite passant par les points (2.14)), coïnside avec la directrice de Wilczynski du faisceau canonique (2.32), il faut et il suffit que l'on ait

$$
\tilde{a}=\left(b_{2}^{0}-b_{3}^{1}\right) / 2 \gamma+\frac{1}{2} \partial \log \gamma / \partial u ; \quad \tilde{b}=\left(a_{1}^{0}-a_{3}^{2}\right) / 2 \beta+\frac{1}{2} \partial \log \beta / \partial v .
$$

On voit ensuite que

$$
a_{3}^{1}-a_{2}^{0}=b_{3}^{2}-b_{1}^{0}
$$

est la condition nécessaire et suffisante, pour que les quadriques $Q_{u}(v)$ et $Q_{u}(v)$ coïncident. On voit également que (5) est la condition pour la coïncidence des quadriques $Q^{\prime}, Q^{\prime \prime}$ de Čech-Lie (cf. [8]).

Si nous choisissons le point $A_{3}$ sur la normale de Fubini dans le faisceau canonique, les équations suivantes doivent être vérifiées

$$
\begin{aligned}
& b_{1}^{1}-b_{2}^{2}-\partial \log \beta / \partial v+\partial \log \left(\beta^{2} \gamma\right) / \partial v=0, \\
& a_{2}^{2}-a_{1}^{1}-\partial \log \gamma / \partial u+\partial \log \left(\beta \gamma^{2}\right) / \partial u=0 .
\end{aligned}
$$

Considérons maintenant la congruence $\Gamma_{1}$ des normales de Fubini. Pour que les surfaces développables de la congruence $\Gamma_{1}$ découpent sur la surface $\pi$ un réseau conjugué, il faut et il suffit que l'on ait

$$
b_{3}^{2}-a_{3}^{1}=0 .
$$


Fixons le point $A_{3}$ sur la normale de Fubini d'une telle manière que $A_{3}$ appartienne: à la surface focale $F_{1}$ de la congruence $\Gamma_{1}$. Alors $A_{3}-2 a_{3}^{1} A_{0}$ est un point de la seconde surface focale $F_{2}$. Le choix du point $A_{3}$, ainsi mentionné, est exprimé analytiquement par la condition

$$
\left(a_{3}^{1}\right)^{2}=b_{3}^{1} a_{3}^{2} .
$$

Si nous demandons maintenant que les deux suriaces focales soient des surfaces: à connexion projective sans torsion (c'est-à-dire que pour chaque couche de courbes $\alpha$ sur la surface donnée la couche conjuguée $\beta$ ait la propriété d'avoir $\alpha$ pour sa. couche conjuguée) nous trouvons par calculs directs que la condition nécessaire et. suffisante pour cela est

$$
a_{3 v}^{1}-b_{3 u}^{1}=b_{3}^{0}-2 \tilde{b} a_{3}^{1}-a_{3}^{2} \gamma, \quad a_{3 v}^{2}-b_{3 u}^{2}=\beta b_{3}^{1}+2 \tilde{a} b_{3}^{2}-a_{3}^{0} .
$$

Soit $\Gamma_{2}$ la congruence de droites qui sont polaires aux droites de la congruence $\Gamma_{1}$ : des normales de Fubini de la surface $\pi$, par rapport à la quadrique de Darboux. (c'est la quadrique que nous obtenons p. ex. de (27) si nous nous rendons compte $\mathrm{du}$ fait que les conditions données ci-dessus sont satisfaites). Pour notre choix du repère, les droites de la congruence $\Gamma_{2}$ sont déterminées par les points $A_{1}, A_{2}$. Sur la droite $\left[A_{1}, A_{2}\right]$ il y a deux points $\lambda_{1} A_{1}+\lambda_{2} A_{2}$ où $\lambda_{1}^{2} a_{1}^{0}-\lambda_{2}^{2} b_{2}^{0}=0$, qui se trouvent. sur les surfaces focales $F_{3}, F_{4}$ de la congruence $\Gamma_{2}$. Les équations

$$
a_{1 v}^{0}-b_{1 u}^{0}=2 \tilde{a} b_{1}^{0}+a_{3}^{0}-\beta b_{2}^{0}, \quad a_{2 v}^{0}-b_{2 u}^{0}=a_{1}^{0} \gamma-b_{3}^{0}-2 b_{1}^{0} \tilde{b}
$$

sont vérifiées si et seulement si les surfaces focales $F_{3}, F_{4}$ sont sans torsion.

Si nous nous rendons compte de ce que (1), (3), (4), (5), (6), (7), (8), (9) ont lieu, le repère étant spécialisé d'après $(2.2),(2.3)$, demandons quelles sont les composantes. $\mathrm{du}$ tenseur (1.17) qui peuvent être différentes de zero. Ce sont les composantes

$$
M_{123}^{0}, M_{121}^{1}, M_{122}^{2} \text {. }
$$

Or nous savons que l'on a $M_{\alpha \beta a}^{b}=-M_{\beta \alpha a}^{b}$, et, dans notre cas, on a $M_{121}^{1}=-M_{122}^{2}$ Nous pouvons donc écrire au lieu de (1.29)

$$
\begin{aligned}
& \stackrel{*}{B_{0}}=A_{0}, \quad \stackrel{*}{B_{1}}=\left(1-r s u^{\beta} v^{\alpha} M_{\alpha \beta 1}^{1}\right) A_{1}, \\
& \stackrel{*}{B_{2}}=\left(1+r s u^{\beta} v^{\alpha} M_{\alpha \beta 1}^{1}\right) A_{2}, \quad \stackrel{*}{B}=-r s u^{\beta} v^{\alpha} M_{\alpha \beta 3}^{0} A_{0}+A_{3} .
\end{aligned}
$$

Les équations (12) expriment une homographie de l'espace local sur lui-même, correspondant à une courbe fermée sur la surface $\pi$. Les équations mentionnées font voir immédiatement que

$$
M_{123}^{0}=0
$$

est la condition nécessaire et suffisante pour que, dans notre cas (où toutes les conditions citées sont supposées satisfaites), la normale de Fubini se transforme en ellemême par une translation parallèle de l'espace local suivant une courbe fermée 
arbitraire sur la surface $\pi$; c'est une correspondance ponctuelle identique, car en tant que droite, l'invariance de la normale de Fubini découle déjà de (12).

Si nous appellons seconde normale de Fubini la droite polaire à la normale de Fubini par rapport à la quadrique de Darboux, alors l'égalité

$$
M_{121}^{1}=0
$$

est la condition nécessaire et suffisante pour que la seconde normale de Fubini se transforme en elle-même, point par point, par une translation parallèle de l'espace local suivant une courbe fermée sur la surface $\pi$. Si toutes les conditions mentionnées ci-dessus sont vérifiées, alors toutes les composantes du tenseur $M_{\alpha \beta a}^{b}$ sont égales à zéro et, en vertu de (1.33) et (1.2), on voit que la surface $\pi$ est unie surface de l'espace projectif. Nous pouvons donc énoncer la proposition suivante:

Les conditions qui vont suivre sont nécessaires et suffisantes dans leur ensemble, pour que la surface $\pi$ à connexion projective soit une surface de l'espace projectif:

a) La surface $\pi$ est sans torsion.

b) La quadrique singulière $Q(v, \lambda)$ dans le faisceau des quadriques de Darboux est le plan tangent à la surface au point considéré, compte deux fois.

c) L'arrête de Green GI coüncide avec l'arrête de Green GII.

d) La directrice de Wilczynski, définie comme sommet d'un complexe spécial est identique avec la directrice de Wilczynski dans le faisceau canonique.

e) Les quadriques $Q_{u}(v), Q_{v}(v)$ (soit resp. les quadriques $Q^{\prime}, Q^{\prime \prime}$ de Čech-Lie) coïncident.

f) Les surfaces développables de la congruence $\Gamma_{1}$ des normales de Fubini découpent sur la surface $\pi$ un réseau conjugué.

g) Les surfaces focales de la congruence $\Gamma_{1}$ des normales de Fubini sont des surfaces à connexion projective sans torsion.

h) Les surfaces focales de la congruence $\Gamma_{2}$ des secondes normales de Fubini sont les surfaces à connexion projective sans torsion.

i) Si les conditions a)-h) sont vérifiées, alors les transformations du groupe d'holonomie (12) conservent les normales de Fubini en tant que droites. La dernière condition i) consiste à supposser que ces droites sont invariantes point par point.

\section{Littérature}

[1] E. Cartan: Leçons sur la théorie des espaces à connexion projective. Paris 1937.

[2] B. Cenkl: Homographies conservant l'élément du troisième ordre d'une surface dans un espace à connexion projective. Czechosl. Math. Journal 12 (87) 1962, 288-293.

[3] B. Cenkl: La normale d'une surface d'espace à connexion projective. Czechosl. Math. Journal $12(87) 1962,582-606$.

[4] B. Cenkl: Les espaces König généralisés. Czechosl. Math. Journal 14 (89) 1964, 1-21.

[5] С. П. Фиников: Проективно-диференциальная геометрия. Москва 1937. 
[6] A. Švec: L'application des variétés à connexion à certains problèmes de la géométrie différentielle. Czechosl. Math. Journal 10 (85) 1960, 523-550.

[7] A. Švec: L'élément linéaire projectiv d'une surface plongée dans l'espace à connexion projective. Czechosl. Math. Journal 8 (83) 1958, 285-291.

[8] A. Švec: Les quadriques de Lie d'une surface plongée dans un espace tridimensionnel à connexion projective. Czechosl. Math. Journal 11 (86) 1961, 134-142.

[9] A. Švec: Sur la géométrie différentielle d'une surface plongée dans un espace à trois dimen-. sions à connexion projective. Czechosl. Math. Journal 11 (86) 1961, 386-397.

\section{Резюме \\ УРАВНЕНИЕ СТРУКТУРЫ ПРОСТРАНСТВА С ПРОЕКТИВНОЙ СВЯЗНОСТЬЮ}

БОГУМИЛ ЦЕНКЛ (Bohumil Cenkl), Прага

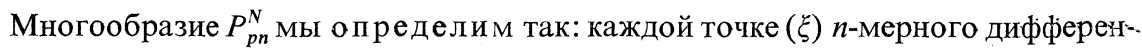
цируемого многообразия $X_{n}$ мы поставим в соответствие $N$-мерное проективное пространство $P_{N}(\xi)$, т. наз. иентр локального пространства. На определенном таким образом многообразии введем связность следующим образом: в локальном пространстве $P_{N}(\xi)$ мы выберем репер $\left\{A_{0}(\xi), \ldots, A_{N}(\xi)\right\}$ так, чтобы $\left\{A_{0}(\xi), \ldots, A_{p}(\xi)\right\}$ было базисом центра $P_{k}(\xi)$ локального пространства $P_{\Lambda}(\xi)$. Тогда связность на многообразии $P_{p n}^{N}$ дана уравнениями (1.1). Условия инте-. грируемости многообразия $P_{p n}^{N}$ с проективной связностью имеют вид (1.2). (1.15) является тензором кривизны кручения указанного многообразия. Уразнением (1.32) дается геометрический смысл обращения его в нуль, а также обращения в нуль его подтензора, т. наз. тензора кручения $R_{\alpha \beta A}^{M}$.

Точке поверхности $\pi$ (многообразия $P_{02}^{3}$ ) можно сопоставить канонический репер, являюшийся инвариантным относительно преобразований локального. пространства $P_{3}(\xi)$ и параметров на поверхности $\pi(2.3)$. Точке поверхности. можно сопоставить инвариантным образом целый ряд геометрических объектов. При помощи этих геометрических объектов в работе установлены необходимые и достаточные условия для того, чтобы поверхность $\pi$ с проективной связностью была поверхностью проективного пространства. 